\title{
MyD88 mediates colorectal cancer cell proliferation, migration and invasion via $\mathrm{NF}-\mathrm{\kappa} \mathrm{B} / \mathrm{AP}-1$ signaling pathway
}

\author{
GUANGWEI ZHU ${ }^{1,2^{*}}$, ZHIBIN CHENG ${ }^{1,2^{*}}$, YONGJIAN HUANG ${ }^{1}$, WEI ZHENG ${ }^{1}$, \\ SHUGANG YANG ${ }^{1}$, CHUNLIN LIN $^{1}$ and JIANXIN YE ${ }^{1,2}$ \\ ${ }^{1}$ Department of Gastrointestinal Surgery 2 Section, The First Hospital Affiliated to Fujian Medical University, \\ Fuzhou, Fujian 350005; ${ }^{2}$ Key Laboratory of Ministry of Education for Gastrointestinal Cancer, \\ Fujian Medical University, Fuzhou, Fujian 350000, P.R. China
}

Received February 9, 2019; Accepted October 15, 2019

DOI: $10.3892 /$ ijmm.2019.4390

\begin{abstract}
The role of myeloid differentiation factor 88 $(M y D 88)$ in malignant tumors is largely unknown. Therefore, in this study, we aimed to examine the function and underlying mechanism of $M y D 88$ in colorectal carcinoma in vitro using SW480 and HCT116 cell lines and in vivo using a nude mouse model. SW480 and HCT116 cells were infected with a lentiviral-based effective $M y D 88$ siRNA virus. CCK- 8 and colony formation assay were used to assess cell proliferation. Transwell and scratch assays were used to test the migration of colorectal cancer cells, and the Transwell assay was further used to analyze the invasiveness of colorectal cancer cells. Western blotting was performed to analyze the underlying mechanism of $M y D 88$ regulation. In vitro experiments demonstrated that silencing MyD88 in SW480 and HCT116 cells markedly suppressed growth and invasion. Furthermore, $M y D 88$ knockdown affected the $M y D 88-N F-\kappa B / A P-1$ signaling pathways in SW480 and HCT116 cells. In vivo, MyD 88 knockdown inhibited tumor growth in a HCT116 cell subcutaneous nude model. We found that knockdown of the $M y D 88$ gene can affect proliferation, invasion, and migration of colorectal cancer cells. We further verified that $M y D 88$ knockdown can reduce the activity of $N F-\kappa B$ and $A P-1$ pathways. These results show that $M y D 88$ gene plays an important role in promoting colorectal cancer, and thus can be exploited as a potential diagnostic and prognostic biomarker for colorectal cancer.
\end{abstract}

Correspondence to: Dr Jianxin Ye, Department of Gastrointestinal Surgery 2 Section, The First Hospital Affiliated to Fujian Medical University, 20th Chazhong Road, Fuzhou, Fujian 350005, P.R. China E-mail: yejianxinfuyi@126.com

*Contributed equally

Key words: myeloid differentiation factor 88, colorectal cancer, proliferation, migration, invasion

\section{Introduction}

Colorectal cancer (CRC) is one of the most common malignant tumors worldwide, with the second highest incidence in men, the third in women, and the third and fourth in cancer-related deaths among men and women (1). Despite recent improvements in screening strategies and the development of more effective CRC treatments, the prognosis for advanced CRC remains poor (2). Many risk factors, including viral and bacterial infections, alcohol, tobacco smoke, aging, ulcerative colitis, and a sedentary lifestyle, as well as genetic mutations may be associated with CRC (3). There are three types of genetic aberrations, namely chromosomal instability (CIN), microsatellite instability (MSI), and $\mathrm{CpG}$ island methylator phenotype (CIMP), involved in the pathogenesis of CRC (4).

Myeloid differentiation factor $88(M y D 88)$ is an essential adaptor molecule for $I L-1$ and Toll-like receptor $(T L R)$ signaling (5). TLRs are a family of pattern recognition receptors that identify various pathogen-associated molecular patterns (PAMPs), molecules derived from various pathogens, and activate host innate immune defense against pathogen invasion. MyD88 signaling plays a predominant role in mediating systemic and cardiac cytokine responses in the survival of activated $\mathrm{CD} 4{ }^{+} \mathrm{T}$ cells to promote tumor cell proliferation, invasion, metastasis and are correlated with the prognosis of HCC patient-mediated inflammatory pathway injury and neurodegenerative tissue injury (6-9). $M y D 88$ expression is an adverse prognostic factor in ovarian cancer and is essential in adenovirus keratitis $(10,11)$. MyD88 is a therapeutic target for inflammatory lung diseases (12). It also contributes to ocular surface homeostasis (13). However, the role of MyD88 in $\mathrm{CRC}$ and the mode of action following its expression remain unknown.

Previous findings showed MyD88 expression in cancer tissue and adjacent normal colorectal tissues of patients with CRC; however, the expression levels were significantly higher in the cancer tissues than in the adjacent tissues (14). The MyD88 expression level was correlated with the clinical stage, $\mathrm{T}$ stage, $\mathrm{M}$ stage and lymph node metastasis, and the survival rate of patients with $\mathrm{CRC}$ and higher $M y D 88$ expression was significantly lower than that of the patients with CRC and lower $M y D 88$ expression. 
The aim of the present study was to determine the role of $M y D 88$ in CRC. The $M y D 88$ gene was knocked down to dissect its functional role in CRC cells. In addition, the mechanism of $M y D 88$ knockdown, which causes change in the related signal pathway, was explored. The findings showed that $M y D 88$ is a crucial factor affecting CRC progression.

\section{Materials and methods}

MyD88 siRNA synthesis and transfection. siRNA target sequences were identified on the human $M y D 88$ sequence. According to the siRNA design guidelines, DNA template oligonucleotides corresponding to three different siRNA sequences (siRNA-1, siRNA-2 and siRNA-3) were designed as follows: siRNA-1: GCCTATCGCTGTTCT TGA A, siRNA-2: GACTGATTCCTATTAAATA, siRNA-3: CAGCGAGCTAATTGAGAAA. These siRNA and negative control (NC) sequences were produced by Genechem Co. Ltd. The SW480 and HCT116 cells were cultured in a medium with $10 \%$ FBS. When these CRC cells were at approximately $90 \%$ confluency, the NC, siRNA-1, siRNA-2 and siRNA-3 sequences were transfected into the cells, using Lipofectamine 3000 (Invitrogen), according to the manufacturer's instructions.

RNA preparation and quantitative PCR amplification. RT-qPCR was used to test mRNA expression of the $M y D 88$ gene. Total RNA was extracted from CRC cell lines, using the Qiagen RNeasy kit (Qiagen Bioinformatics) according to the manufacturer's instructions, and quantified using UV260/280 $\mathrm{nm}$ to an absorption ratio of z1.8. An RT Reagent kit (Takara Bio Lnc.) was used for reverse transcription of RNA into cDNA. The primers (BioSune Biotechnology Co., Ltd.) $M y D 88$ F: GGCTGCTCTCAACATGCGA, R: CTG TGTCCGCACGTTCAAGA and GAPDH F: GAAGGTGAA GGTCGGAGTC, R: GAAGATGGTGATGGGATTTC, were designed to amplify cDNA with SYBR Premix EX Taq kit (Takara Bio Lnc.). PCR conditions were $95^{\circ} \mathrm{C}$ for $2 \mathrm{~min}, 95^{\circ} \mathrm{C}$ for $15 \mathrm{sec}$, and $60^{\circ} \mathrm{C}$ for $30 \mathrm{sec}$ for 40 cycles. The relative amount of MyD88 mRNA was normalized to that of GAPDH. The $2^{-\Delta \Delta \mathrm{Cq}}$ method was used to calculate this as Livak and Schmittgen had reported (15).

Construction of lentiviral vectors. In order to establish better stable transfection for later experiments, we constructed lentiviral-based siRNA targeting $M y D 88$ vector. The primers, designed by BioSune Biotechnology Co., Ltd. contained two restriction sites, AgeI (underlined) and EcoRI (in bold) (siRNA1 F: CCGGGCCTATCGCTGTTCTTGAATTCA AGAGATTCAAGAACAGCGATAGGCTTTTTTGGTACC, R: AATTGGTACCAAAAA AGCCTATCGCTGTTCTTG AATCTCTTGAATTCAAGAACAGCGATAGGC; siRNA2 F: CCGGGACTGATTCCTATTAAATATTCA AGAGAT ATtTAatAGgAatCAGTCTTTTTTGGTACC, R: AAT TGGTACCAAAAAAGACTGATTCCTATTAAATATCTC TTGAATATTTAATAGGAATCAGTC; and siRNA3 F: $\underline{\text { CG }}$ GCAGCGAGCTAATTGAGAAATTCAAGAGATTTCTCA ATTAGCTCGCTGTTTTTTGGTACC, R: AATTGGTAC CAAAAAACAGCGAGCTAATTGAGAAATCTCTTGAAT TTCTCAATTAGCTCGCTG). The primers were designed to anneal and form double-stranded DNA. pLKO.1-EGFP-Puro (Fenghui Bio) was used as an empty vector for purification and recovery (Tiangen) after double digestion with AgeI and EcoRI (Thermo Fisher Scientific, Inc.). The purified linear empty vector and the double-stranded DNA formed by annealing were then ligated with T4 DNA Ligase (Invitrogen) and transformed, using stabl3, cloned, sequenced, and then subjected to plasmid extraction (Tiangen) for the next experiment.

Lentiviral infection and stable cell line screening. The SW480 and HCT116 cell lines were obtained from the cell bank of the Chinese Academy of Sciences (Shanghai, China). The two cell lines were cultured and maintained in DMEM (Gibco BRL) with $10 \%$ fetal bovine serum (FBS), and incubated at $37^{\circ} \mathrm{C}$ and $5 \% \mathrm{CO}_{2}$. The validated $M y D 88$ knockdown plasmid and empty vector were co-transfected with pMD2.G and psPAX2 (Fenghui Bio) into 293T cells, and the supernatant was filtered after 2 days of culture. HCT116 and SW480 cells were infected with the supernatant, and after 2 weeks of screening with puromycin, the protein was extracted for verification. Stably expressed cells were selected for subsequent experiments.

Western blot analysis. Western and IP cell lysis buffer (Beyotime), containing 1\% PMSF (Amresco), was used to cleave proteins for $30 \mathrm{~min}$ on ice. After $30 \mathrm{~min}$, the lysed cells were centrifuged at $12,000 \mathrm{x} \mathrm{g}$ for $10 \mathrm{~min}$ at $4^{\circ} \mathrm{C}$ to extract the supernatant for protein quantification using the BCA Protein Assay kit (Thermo Fisher Scientific, Inc.), and boiled for $10 \mathrm{~min}$ by adding $5 \mathrm{X}$ SDS. The total amount of protein $(50 \mu \mathrm{g})$ was added to the prepared $12 \%$ SDS-PAGE gels for electrophoretic separation and transferred to $0.45 \mu \mathrm{m}$ PVDF membranes (Amersham Hybond, GE Healthcare). The membranes were then blocked with $1 \%$ albumin from bovine serum (Amresco) for $2 \mathrm{~h}$. Next, the membranes were incubated overnight with diluted $M y D 88$ (1:1,000, AF5195; Affinity), GAPDH (1:1,000, ab181602; Abcam), NF- $\kappa B$ p65 (1:1,000, AF5006; Affinity), $p-N F-\kappa B$ p65 (1:1,000, \#3033; Cell Signaling), $c$-jun (1:1,000, bs-0670R; Bioss), and $p$-c-jun $\left(1: 1,000\right.$, bs-3172R; Bioss) antibodies on a shaker at $4^{\circ} \mathrm{C}$. The membranes were washed for 10 min three times with TBS-T (0.1\% Tween-20) at room temperature, incubated in goat anti-rabbit IgG H\&L (HRP) (1:2,000, ab7090; Abcam) for $1 \mathrm{~h}$, and then washed. Subsequently the membranes were exposed to enhanced chemiluminescence substrate detection solution (Lulong Biotech).

Colony formation assay. Cells (500/well) were seeded in a $6-\mathrm{cm}$ plate, and cultured for 14 days in a culture medium, containing $10 \%$ FBS. The culture solution was discarded and cells were infiltrated with methanol for $10 \mathrm{~min}$, stained with crystal violet, washed with water, dried and counted.

Cell proliferation assay. CCK-8 (Dojindo) was used to detect cell proliferation. Cells were seeded, at densities of 1,000 cells/well, in a 96-well plate, then incubated at $37^{\circ} \mathrm{C}, 5 \% \mathrm{CO}_{2}$. After 24,48 , 72 , and $96 \mathrm{~h}$, the culture solution was discarded and $100 \mu \mathrm{l}$ of $10 \%$ CCK-8 serum-free medium was added. Cell proliferation was estimated using a microplate reader (Bio-Tek) after $1 \mathrm{~h}$ of incubation.

Cell migration and invasion assay. In the migration experiment, $4 \times 10^{4} \mathrm{CRC}$ cells, in serum-free medium, were seeded 


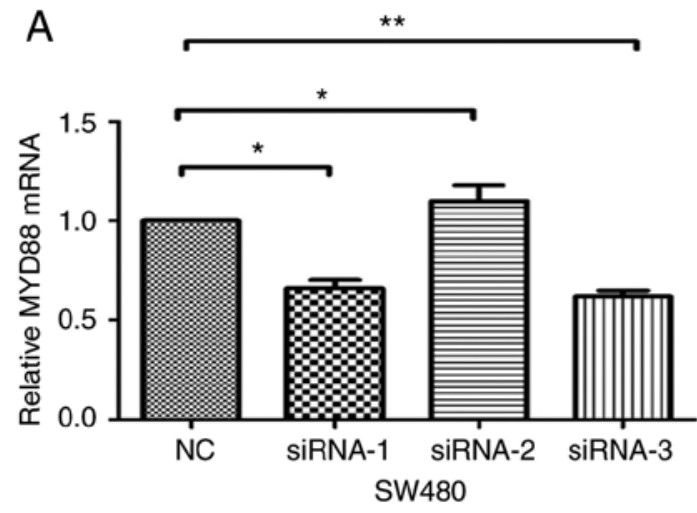

B

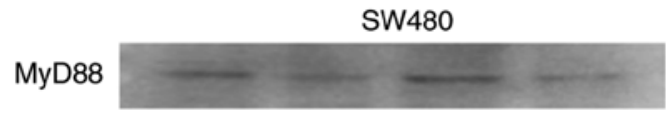

GAPDH

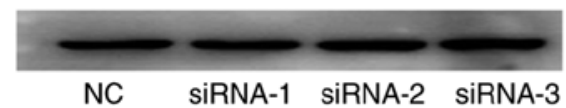

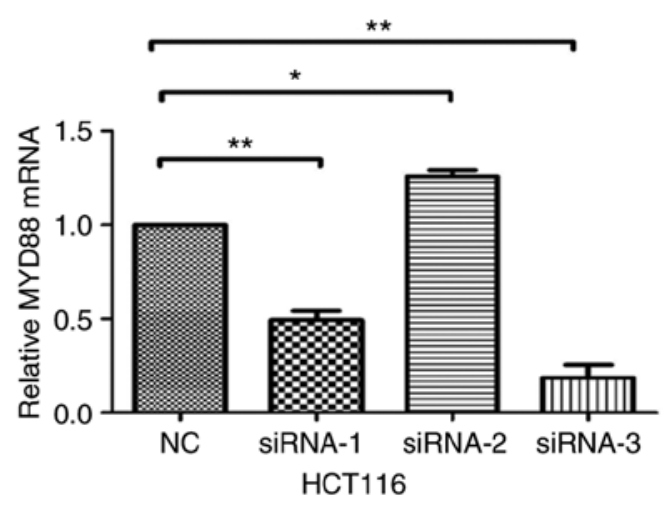

HCT116

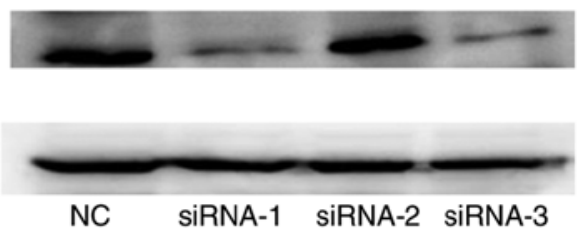

C
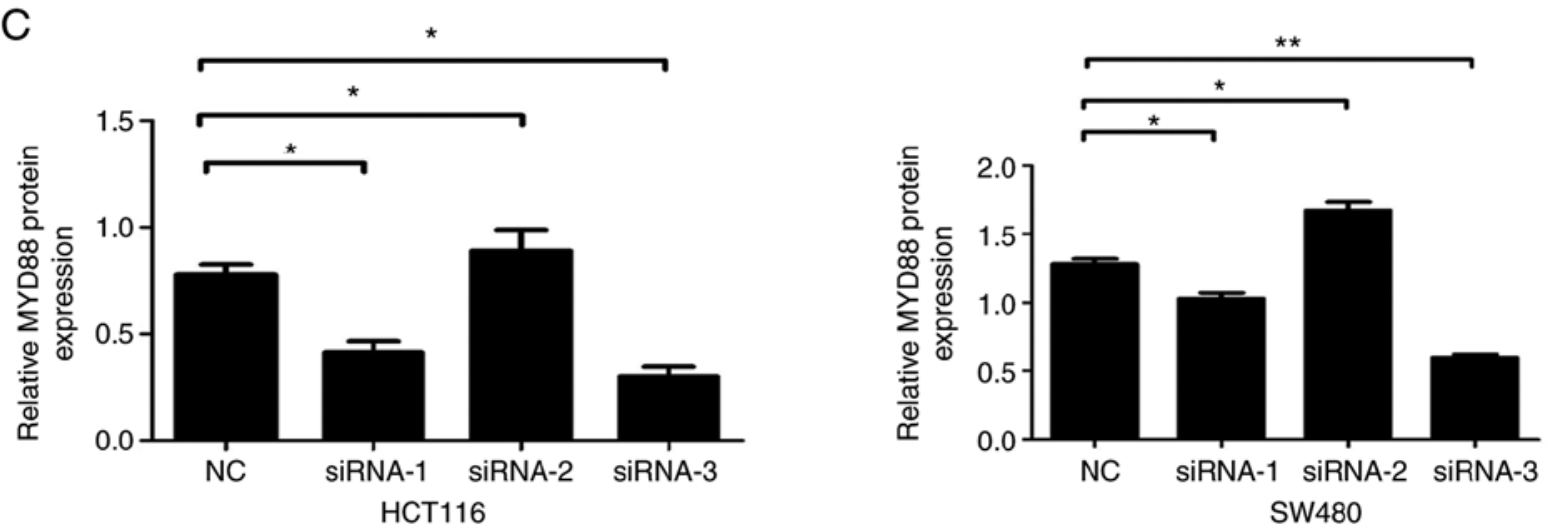

Figure 1. Knockdown of MyD88 in the SW480 and HCT116 cells. (A) Total RNA from SW480 and HCT116 cells after transfection using NC, siRNA-1, siRNA- 2 and siRNA-3 sequences were assessed by RT-qPCR using $M y D 88$ primers. GAPDH was amplified for an internal control. The siRNA-1 and siRNA-3 sequences resulted in higher suppression of the levels of MyD88 mRNA. (B) MyD88 protein in the SW480 and HCT116 cells after transfection using NC, siRNA-1, siRNA-2 and siRNA-3 sequences were detected by western blotting. GAPDH protein was used an internal control. (C) Semi-quantitative analysis showed that $M y D 88$ protein levels in siRNA-1 and siRNA-3 cells were markedly lower compared with NC and siRNA-2 cells. The mRNA expression of MyD88 in SW480 NC group and HCT116 NC group cells were taken as 1. Error bars represent mean $\pm \mathrm{SEM}$, representative of three experiments, ${ }^{*} \mathrm{P}<0.05,{ }^{* *} \mathrm{P}<0.01$.

into the upper chamber of a Transwell insert (8-mm pore size; Corning Inc.), and a medium with $20 \%$ FBS was added in the lower chamber as a chemoattractant. In the invasion experiment, Matrigel (BD Bioscience) was coated on the upper chamber seeded with $9 \times 10^{4}$ cells, and the lower chamber contained $20 \% \mathrm{FBS}$ medium. After incubation at $37^{\circ} \mathrm{C}, 5 \% \mathrm{CO}_{2}$ for $48 \mathrm{~h}$, the Transwell chamber was taken out and the medium in the well was discarded and washed with calcium-free PBS. The cells were, then, fixed with methanol for $30 \mathrm{~min}$ and stained with $0.1 \%$ crystal violet for $20 \mathrm{~min}$. The upper unmigrated cells were gently wiped off with a cotton swab, and counted under microscope.

Wound healing assay for CRC cell migration. The cells were seeded in a 6-well plate. After the cells had grown to $100 \%$ confluency, the cell layer was scratched with a $20 \mu$ l pipette tip and the medium containing $10 \%$ FBS was replaced with a serum-free medium. Images of the cells were captured at 0 , 24,48 , and $72 \mathrm{~h}$.
Animal experiments. The experiment performed with animals was approved by the Institutional Animal Care and Use Committee at the Fujian Medical University. Ten five-week-old BALB/c nude mice raised at the Animal Experimental Center of Fujian Medical University were used in this study. HCT116-pLKO.1 or HCT116-pLKO.1-sh3 cells $\left(2 \times 10^{6}\right)$, in serum-free DMEM medium, were injected into the bilateral subcutaneous (HCT116-pLKO.1 cells on one side and HCT116-pLKO.1-sh3 cells on the other side) of 10 mice. The manipulation was stable, rapid and gentle to reduce discomfort in mice. During tumor growth, the tumor volume was measured every four days using the formula: length $\mathrm{x}$ width ${ }^{2} / 2$. and the correlation function graph was plotted. After 6 weeks of tumor growth the nude mice were euthanized by carbon dioxide $\left(100 \% \mathrm{CO}_{2}\right.$ gas replacement rate at $10-30 \%$ container volume/min), and the tumors were removed after the heartbeat and respiratory arrest of nude mice, photographed and immediately weighed. The tumors were soaked in formalin for immunohistochemical analysis. 
A

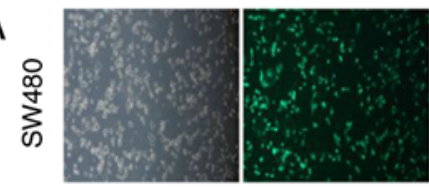

pLKO.1

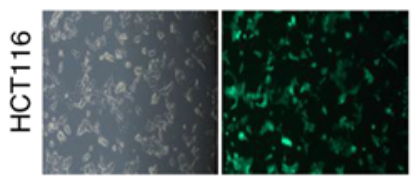

pLKO.1

B
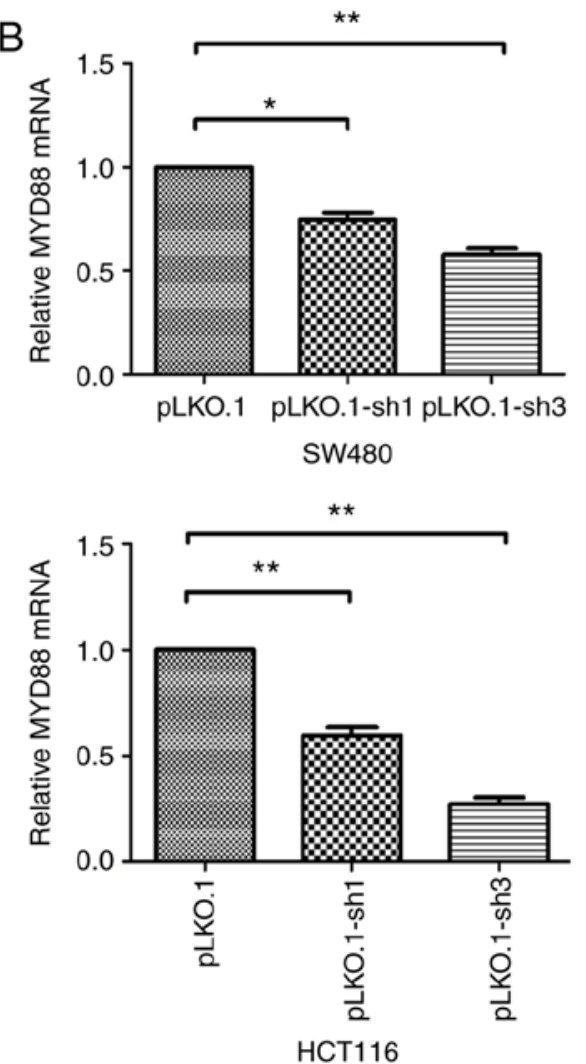

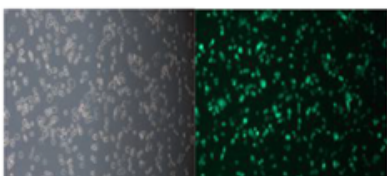

pLKO.1-sh1

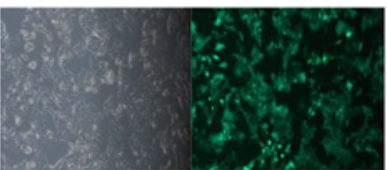

pLKO.1-sh1

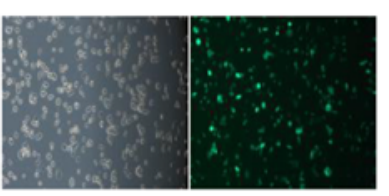

pLKO.1-sh3

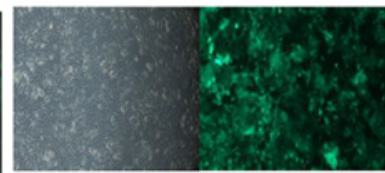

pLKO.1-sh3
C

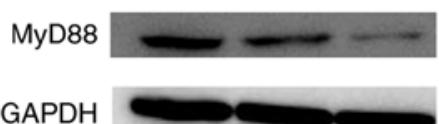

HCT116

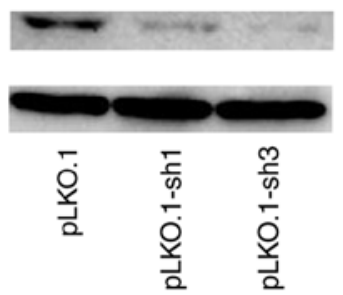

D

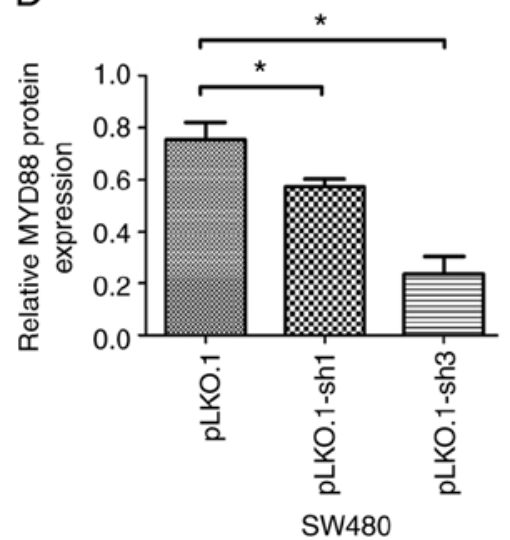

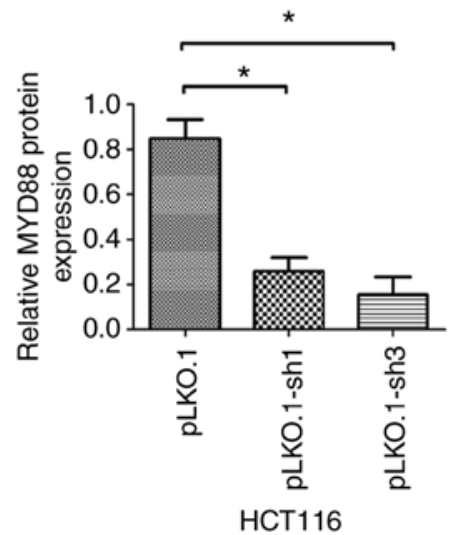

Figure 2. Downregulation of $M y D 88$ mRNA and protein expression by lentivirus-mediated $M y D 88$ siRNA in SW480 and HCT116 cells. (A) pLKO.1, pLKO.1-sh1 and pLKO.1-sh3 lentivirus infected the SW480 and HCT116 cells. After $72 \mathrm{~h}$ infection, the green fluorescent protein (GFP) as demonstrated by fluorescence microscopy. (B) The mRNA and (C) protein expression was analyzed using RT-qPCR and western blot analysis after infection with lentivirus in the SW480 and HCT116 cells. GAPDH was used as an internal control. (D) Semi-quantitative analysis showed that the level of $M y D 88$ protein were significantly inhibited by pLKO.1-sh1 and pLKO.1-sh3 in the SW480 and HCT116 cells. The mRNA expression of MyD88 in SW480 pLKO.1 group and HCT116 pLKO.1 group cells were taken as 1 . Error bars represent mean $\pm \mathrm{SEM}$, representative of three experiments. ${ }^{*} \mathrm{P}<0.05$, ${ }^{* *} \mathrm{P}<0.01$.

Immunohistochemistry. Immunohistochemistry was performed on formalin-fixed, paraffin-embedded tumor tissue specimens from the mice. After dewaxing, hydration, and antigen retrieval, the remaining experimental procedures were performed in accordance with the UltraSensitive ${ }^{\mathrm{TM}} \mathrm{SP}$ (mouse/rabbit) IHC kit instructions. Finally, the degree of staining of the slices was observed under the microscope after DAB staining, hematoxylin counterstaining and neutral resin sealing.

Statistical analysis. Statistical analyses were performed using GraphPad Prism 5 software. The data were expressed as the means \pm standard deviation (SD) and analyzed by one-way ANOVA or Student's t-test. Differences with P-values $<0.05$ were considered to be statistically significant.

\section{Results}

Expression of MyD88 mRNA and protein after siRNA transfection. An RNAi-based method was employed to silence $M y D 88$ and detect the effects of knocking down the gene. NC, siRNA-1, siRNA-2 and siRNA-3 sequences were transfected into SW480 and HCT116 cells. The MyD88 mRNA and protein levels, determined $48 \mathrm{~h}$ after transfection using RT-qPCR and western blot analysis, were shown to decrease in the siRNA-transfected SW480 and HCT116 cells. Compared with NCsiRNA and siRNA-2, which had no effect on MyD 88 mRNA and protein expression levels, siRNA-1 and siRNA-3 sequences inhibited MyD 88 mRNA and protein expression levels (Fig. 1A-C). Since siRNA-1 and siRNA-3 were the most effective siRNA, and these sequences were selected to silence $M y D 88$ in this study. 
A
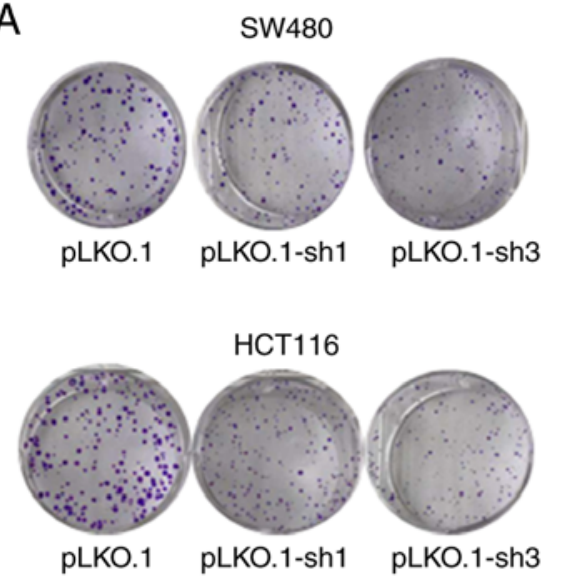

C

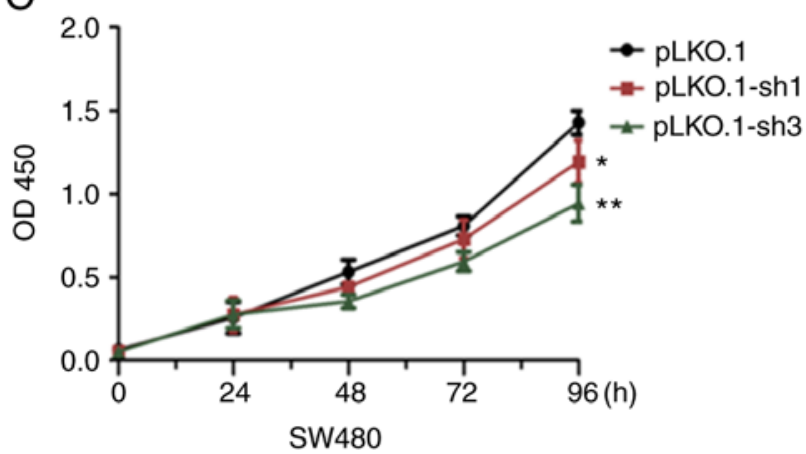

B

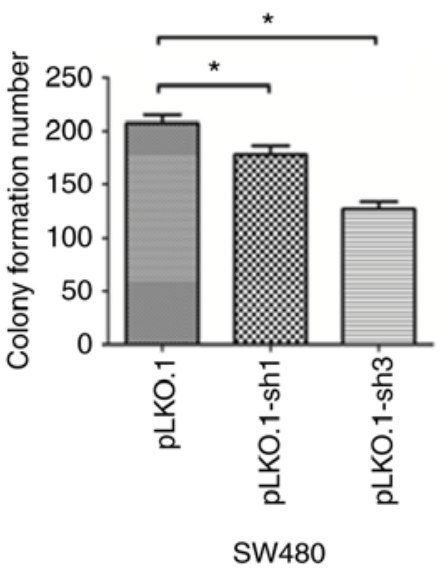

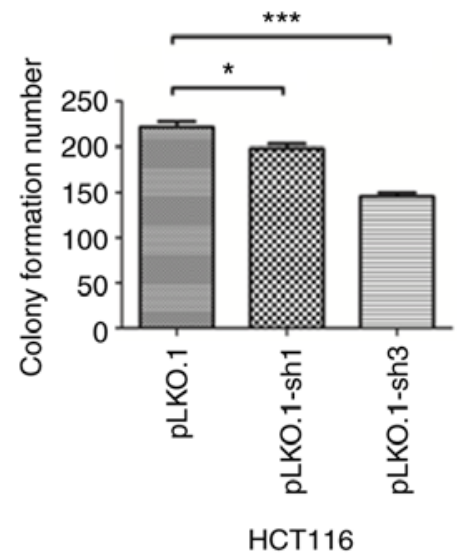

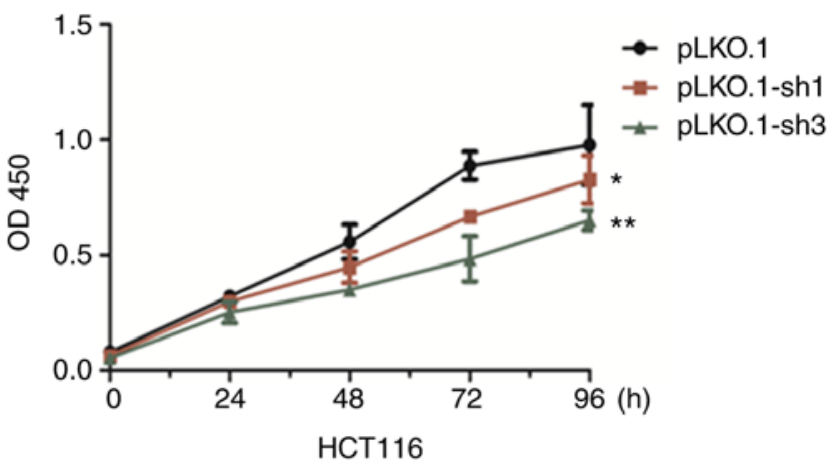

Figure 3. Effects of siMyD88 on the viability of pLKO.1, pLKO.1-sh1 and pLKO.1-sh3 in the SW480 and HCT116 cells. (A and B) Downregulation of MyD88 reduced the colony number in colony formation assays in the SW480 and HCT116 cells. (C) CCK- 8 assays revealing that $M y D 88$ knock-down inhibits the proliferation of SW480 and HCT116 cells. Error bars represent mean $\pm \mathrm{SEM}$, representative of three experiments. ${ }^{*} \mathrm{P}<0.05,{ }^{* * *} \mathrm{P}<0.01,{ }^{* * * *} \mathrm{P}<0.001$.

Expression of MyD88 $\mathrm{mRNA}$ and protein after infection with pLKO.1-sh1 and pLKO.1-sh3 lentiviral vectors. The NC, siRNA-1 and siRNA-3 sequences were constructed into lentiviral vectors and packaged as lentiviruses carrying $\mathrm{NC}$, siRNA-1 and siRNA-2 sequences named pLKO.1, pLKO.1-sh1 and pLKO.1-sh3, respectively. Infection efficiency was quantified by counting cells under a fluorescence microscope $48 \mathrm{~h}$ after infection, because the pLKO.1 vector can express green fluorescent protein in the SW480 and HCT116 cells, which had $>95 \%$ efficiency (Fig. 2A). Quantitative PCR and western blot analyses showed that MyD $88 \mathrm{mRNA}$ and protein levels were markedly inhibited after infection in SW480 and HCT116 cells. Compared with the pLKO.1 group, the pLKO.1-sh1 and pLKO.1-sh3 groups revealed relatively lower $M y D 88$ mRNA expression levels in SW480 and HCT116 cells (Fig. 2B). Consistent with mRNA expression, western blotting showed inhibition of $M y D 88$ protein expression in the pLKO.1-sh1 and pLKO.1-sh3 groups compared with pLKO.1 group in SW480 and HCT116 cells (Fig. 2C and D). To generate stably transfected pLKO.1, the pLKO.1-sh1 and pLKO.1-sh3 cells, the culture medium was replaced with a selection medium containing $2 \mu \mathrm{g} / \mathrm{ml}$ puromycin (Sigma) for two weeks. Stably transfected CRC cells were cultured using medium containing $0.5 \mu \mathrm{g} / \mathrm{ml}$ puromycin. These cells were then used for subsequent experiments.

Knockdown of MyD88 reduces the proliferation of $S W 480$ and HCT116 cells. To verify whether the expression of MyD88 can affect tumor proliferation, stably transfected pLKO.1, the pLKO.1-sh1 and pLKO.1-sh3 CRC cells were used. Cell proliferation was estimated using colony formation and CCK-8 assays. The colony formation assay showed a decrease $(\mathrm{P}<0.05)$ in the number of colonies in the pLKO.1-sh1 and pLKO.1-sh3 groups, while there was no significant difference in the colony numbers in the pLKO.1-sh1 and pLKO.1-sh3 groups $(\mathrm{P}>0.05)$, when compared to the pLKO.1 group in the SW480 and HCT116 cells (Fig. 3A and B). CCK-8 experiment results showed that compared to the LKO.1 group, cell proliferation of the pLKO.1-sh1 and pLKO.1-sh3 groups, which were similar, was lower in the SW480 and HCT116 cells $(\mathrm{P}<0.05)$ (Fig. 3C). The cell viabilities of the pLKO.1-sh1 and pLKO.1-sh3 groups were decreased when compared with the pLKO.1 group in both colorectal carcinoma cell lines. These results indicate that silencing $M y D 88$ contributes to the inhibition of CRC cell proliferation.

MyD88 knockdown attenuates migration and invasion of colorectal carcinoma cells. To determine the ability of $M y D 88$ knockdown in colorectal carcinoma cells to suppress migration, the Transwell assay and scratch test were used to verify the migration ability of SW480 and HCT116 cells harboring MyD88 knockdown. Similar to migration, the invasiveness of the SW480 and HCT116 cells was tested using the Transwell assay. Wound healing/scratch was used to confirm changes in cell migration by observing the extent of wound closure. In the pLKO.1-sh1 and pLKO.1-sh3 groups $(\mathrm{P}>0.05)$, the healing 
A

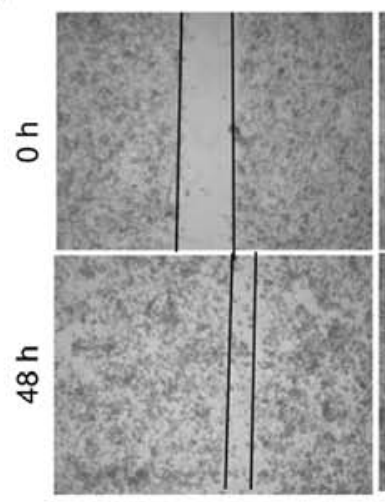

pLKO.1
SW480

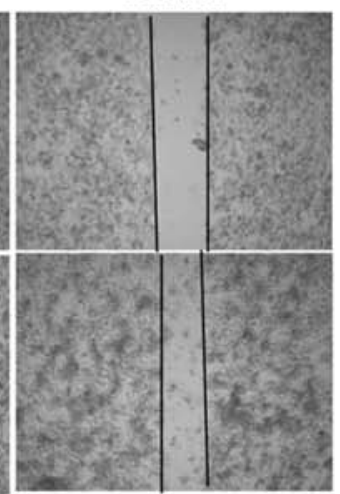

pLKO.1-sh1
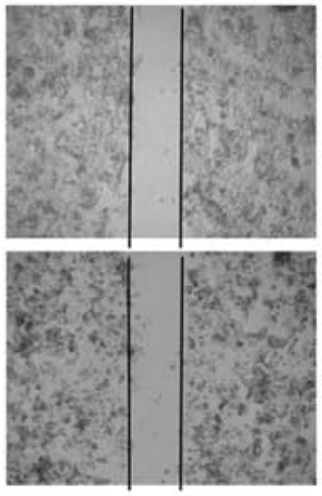

pLKO.1-sh3

C

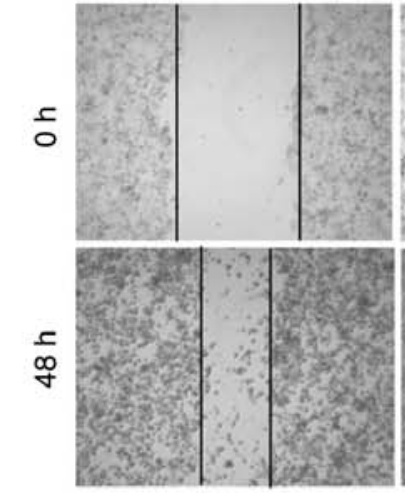

pLKO.1

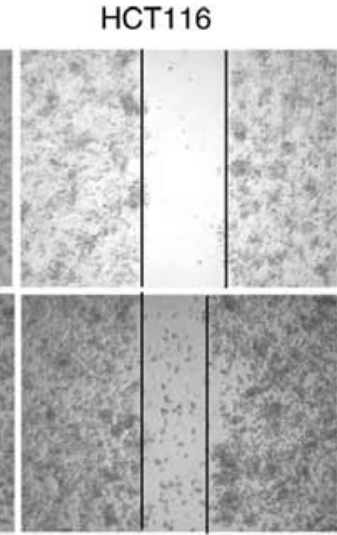

pLKO.1-sh1

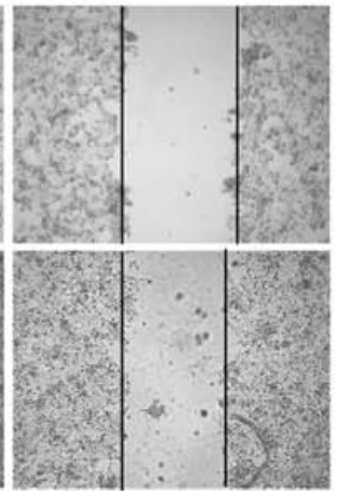

pLKO.1-sh3
B

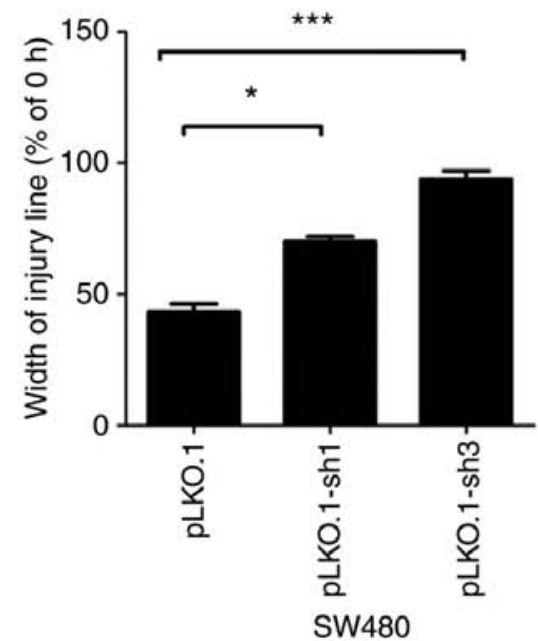

D

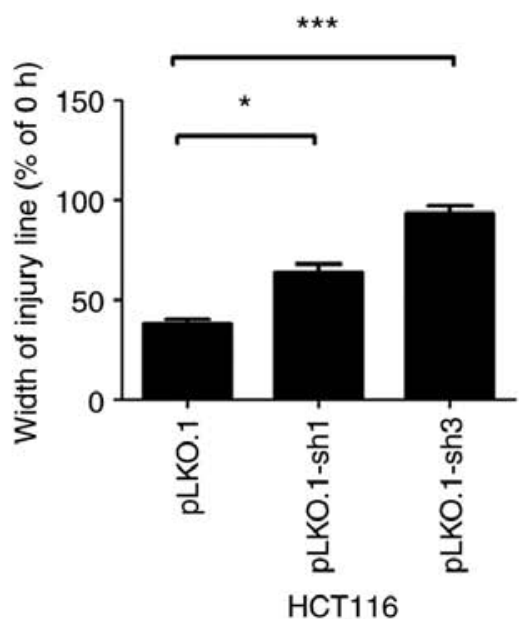

Figure 4. Effects of siMyD88 on the migration of pLKO.1, pLKO.1-sh1 and pLKO.1-sh3 in the SW480 and HCT116 cells. (A) In the SW480 cells, representative images of pLKO.1, pLKO.1-sh1 and pLKO.1-sh3 cell wound healing and microscopic observations were photographed 0 and $48 \mathrm{~h}$ after scratching the cell surface. (B) The width of the scratch was analyzed using a histogram in the SW480 cells. (C) In the HCT116 cells, representative images of pLKO.1, pLKO.1-sh1 and pLKO.1-sh 3 cells wound healing and microscopic observations were photographed 0 and $48 \mathrm{~h}$ after scratching the cell surface. (D) The width of the scratch was analyzed using a histogram in the HCT116 cells. Error bars represent mean \pm SEM, representative of three experiments. ${ }^{*} \mathrm{P}<0.05,{ }^{* * * *} \mathrm{P}<0.001$.

speed of the scratches were lower than in the pLKO.1 group in SW480 and HCT116 cells $(\mathrm{P}<0.05)$ (Fig. 4A-D). To further verify the results of the migration, we used the Transwell experiment. The number of colorectal cells in the pLKO.1 group that migrated through the Transwell polycarbonate filter was markedly higher than that of cells in the pLKO.1-sh1 group $(\mathrm{P}<0.05)$, which was similar to the number of cells in the pLKO.1-sh3 group in the SW480 and HCT116 cells $(\mathrm{P}>0.05)$ (Fig. 5A and B). The results showed that knockdown of $M y D 88$ significantly suppressed cell migration ability compared with the NC group. In vitro cell invasion showed that the number of cells penetrating the basement membrane was significantly higher in the pLKO.1 group than in the pLKO.1-sh1 and pLKO.1-sh3 groups $(\mathrm{P}>0.05)$ in the SW480 and HCT116 cells (Fig. 5C and D).

MyD88 affects $N F-\kappa B$ and AP-1 signaling pathways. In the above experiments, we confirmed that $M y D 88$ can affect the biological behavior of CRC cells, but the underlying mechanisms remain unknown. To investigate the mechanism by which $M y D 88$ silencing leads to a decrease in proliferation and invasion, we evaluated $N F-\kappa B(p 65), p-N F-\kappa B(p-p 65)$, $A P-1$ ( $c$-jun) and $p$ - $A P-1$ ( $p c$-Jun) proteins; these proteins are critical for the growth and invasion of cancer cells, and are very important for $N F-\kappa B$ and $A P-1$ signaling pathways. Western blot analysis showed that $N F-\kappa B(p 65), p-N F-\kappa B(p-p 65), A P-1$ ( $c$-jun) and $p-A P-1$ ( $p$ - $c$-jun) protein levels in the pLKO.1 group cells increased compared with those in the pLKO.1-sh1 and pLKO.1-sh3 groups $(\mathrm{P}<0.05)$ in the SW480 and HCT116 cells (Fig. 6A-D). The results indicated that MYD 88 suppressed the expression of related signaling pathway proteins.

Knockdown of MyD88 suppresses tumor growth in BALB/c nude mice with subcutaneous xenograft tumors. To verify whether tumor growth in vivo was the same as in vitro, the HCT116-pLKO.1 or HCT116-pLKO.1-sh3 cells were subcutaneously xenografted into 5-week-old BALB/c nude mice (5 mice/group). At 6 weeks of subcutaneous tumor growth, nude mice were all successfully established in a subcutaneous mouse model (Fig. 7A). At autopsy, none of the mice had any ascites or liver, lung or lymph node metastasis. The results showed that the growth rate of subcutaneous tumors in the 

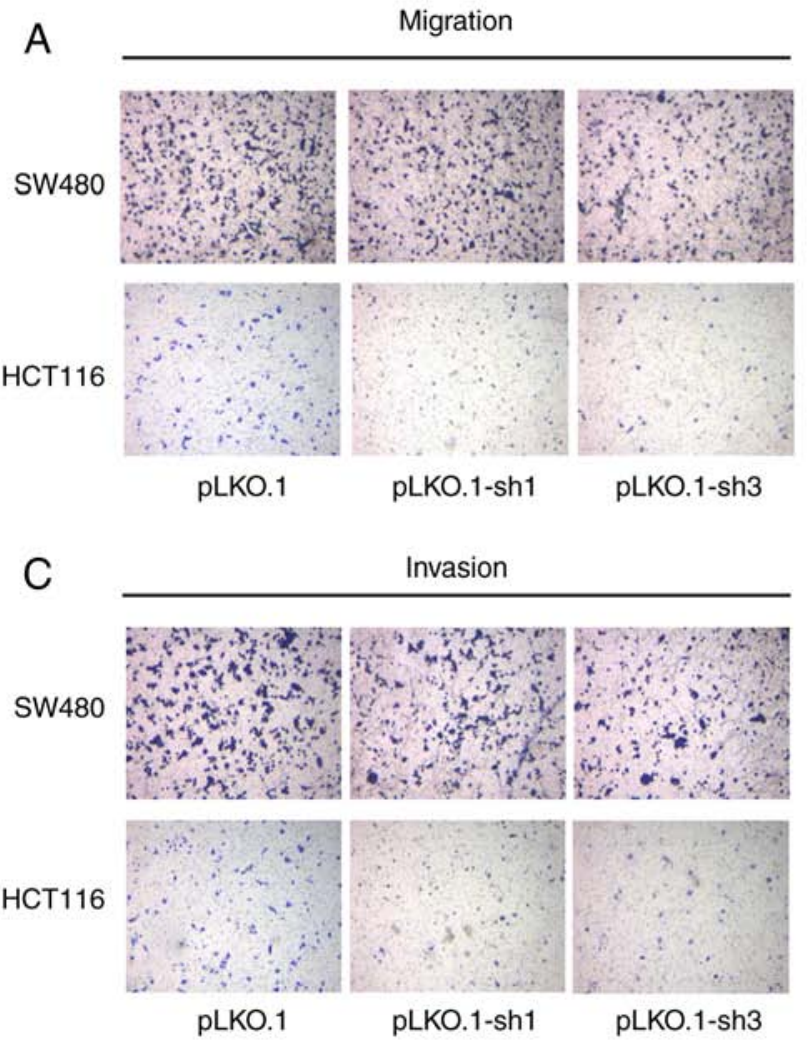

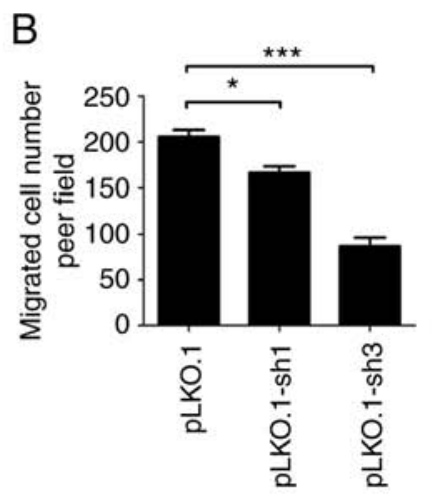

SW480

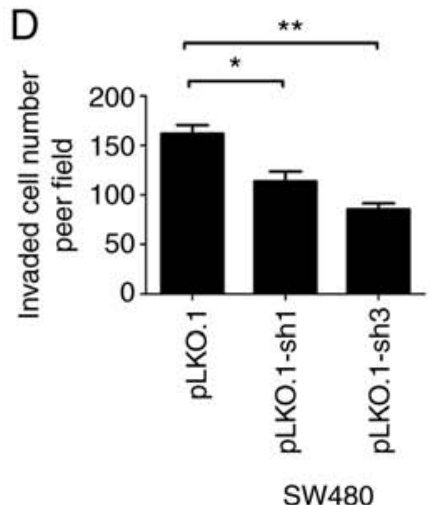

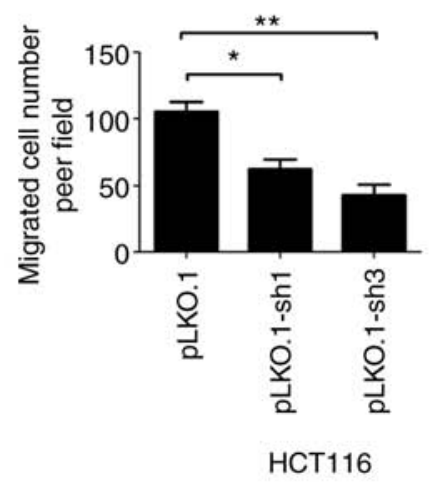

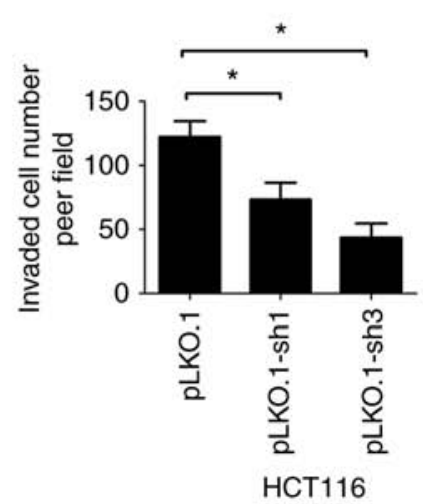

Figure 5. Effects of $s i M y D 88$ on SW480 and HCT116 cells migration and invasion. (A) Migration ability of pLKO.1, pLKO.1-sh1 and pLKO.1-sh3 in the SW480 and HCT116 cells. (B) Number of migrated cells in the pLKO.1, pLKO.1-sh1 and pLKO.1-sh3 groups in the SW480 and HCT116 cells. (C) Invasion ability of pLKO.1, pLKO.1-sh1 and pLKO.1-sh3 in the SW480 and HCT116 cells. (D) Number of invaded cells in the pLKO.1, pLKO.1-sh1 and pLKO.1-sh3 groups in the SW480 and HCT116 cells. Error bars represent mean \pm SEM, representative of three experiments. ${ }^{*} \mathrm{P}<0.05,{ }^{* * *} \mathrm{P}<0.01,{ }^{* * *} \mathrm{P}<0.001$.

HCT116-pLKO.1-sh3 group was significantly lower than that in the HCT116-pLKO.1 group ( $\mathrm{P}<0.05)$ (Fig. 7B). In addition, the tumor weight of the HCT116-pLKO.1-sh3 group was markedly lower than that of the HCT116-pLKO.1 group $(\mathrm{P}<0.05)$ (Fig. 7C). Since $M y D 88$ protein expression was inhibited in HCT116-pLKO.1-sh3 cells, immunohistochemistry was used to analyze the expression levels of MyD88 in subcutaneous xenograft tumors. Compared with the HCT116-pLKO.1 group, MyD88 expression levels were markedly inhibited in the HCT116-pLKO.1-sh3 group $(\mathrm{P}<0.05)$ (Fig. 7D and E).

\section{Discussion}

MyD88 plays a central role in innate immune response. It regulates $N F-\kappa B$ signaling activity (16) and the MAPKs-c-jun (AP-1) signaling pathway (17). MyD88 and MyD88-related signaling have been shown to be involved in the progression and carcinogenesis of cancer-associated cells, with both intrinsic and extrinsic inflammation. In addition, detection of abnormal MyD88 expression is used to predict the prognosis of various human cancers (e.g., lymph, liver) (18). Hence, $M y D 88$ can be considered a potential carcinogenic marker for related research. It will have a positive impact on the pathogenic factors and treatment of CRC.

In the current study, we successfully knocked down the $M y D 88$ gene and verified the knockdown effect of the protein using cell fluorescence, RT-qPCR and western blot analysis.
We determined whether $M y D 88$ gene was able to affect the biological behavior of CRC cells, and subsequently confirmed the ability of $M y D 88$ knockdown to inhibit cell proliferation and invasion in vivo using relevant cell function tests. The results suggest that knockdown MyD88 can suppress proliferation, invasion, and migration of CRC. These findings agree with those of previous studies which showed that, the SNP of $M y D 88$ was associated with poor survival of Caucasian patients with CRC (19). Ikebe et al (20) reported that $s i M y D 88$ can decrease the invasiveness and migration ability of pancreatic cancer cells. Wang et al (21) indicated that a high $M y D 88$ expression is associated with liver metastasis and poor prognosis in CRC patients. We also found that $M y D 88$ expression was high in CRC tissues and was associated with poor survival in colorectal carcinoma patients (14).

Similarly, we explored whether knockdown of MyD 88 gene had the same effect in vitro. We used subcutaneous injection of colon cancer cells to conduct subcutaneous tumorigenesis experiments, confirming that knockdown of $M y D 88$ gene in mice can also inhibit tumor growth. This finding is in agreement with previous studies, which considered that $M y D 88$ increased the risk of developing colorectal neoplasm $(22,23)$. Our findings indicate that MyD88 can promote the growth of CRC. Thus, knockdown of $M y D 88$ gene can inhibit tumor growth, invasion, migration and other related behavioral changes.

However, the specific mechanism by which MyD 88 induces biological behavior in CRC is unclear. Previous 
A
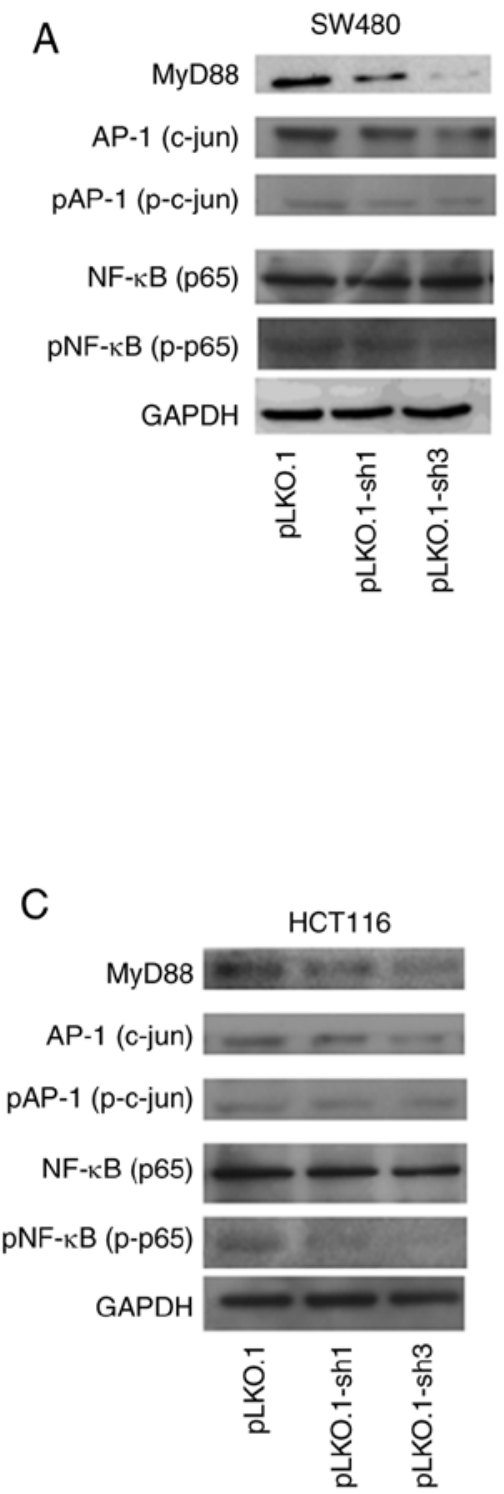

B
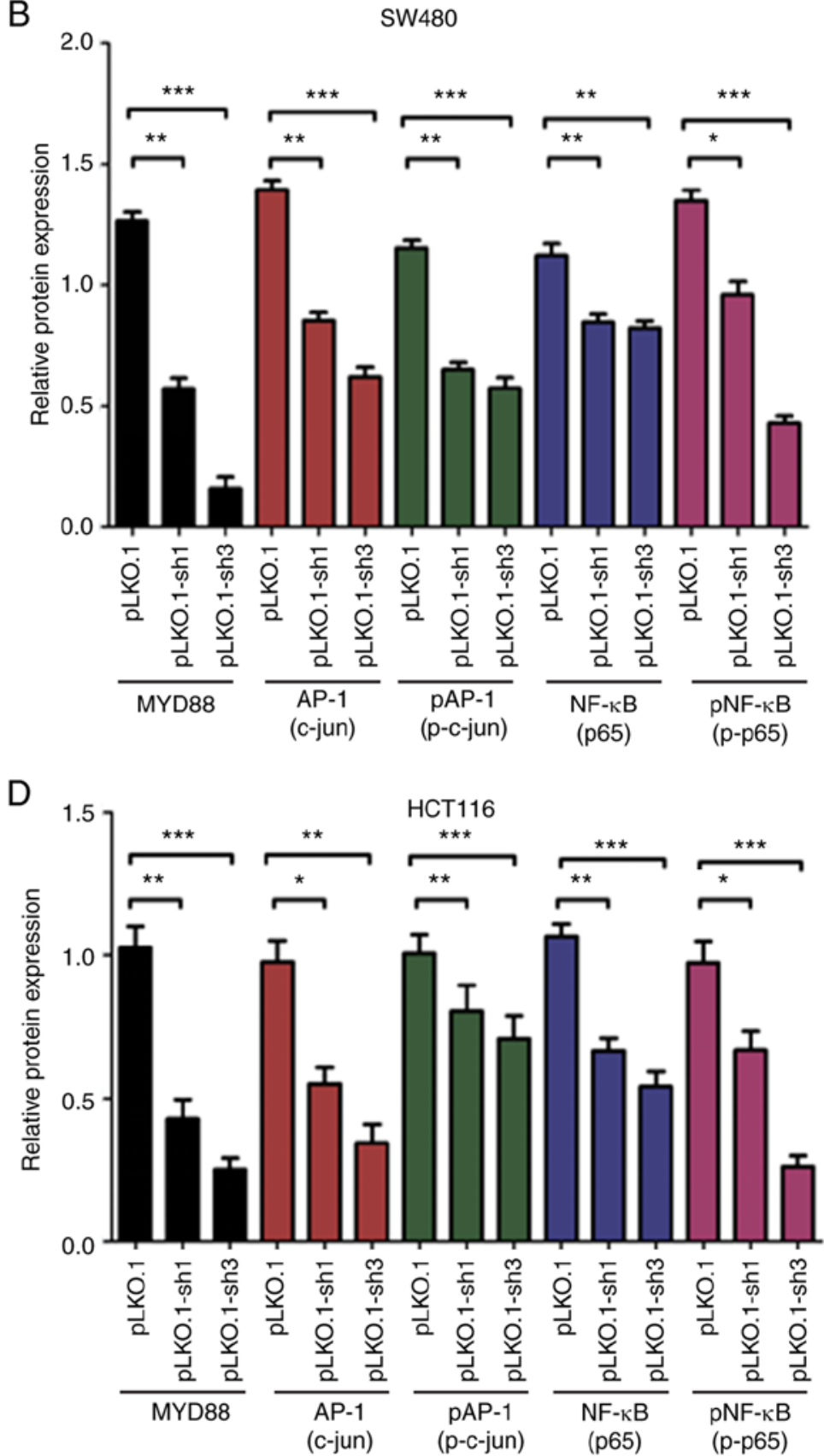

Figure 6. Western blot analysis revealed that the silencing of $M y D 88$ markedly inhibited the expression $N F-\kappa B(p 65), p-N F-\kappa B(p-p 65), A P-1$ (c-jun) and $p-A P-1$ ( $p$-c-jun) protein in the SW480 and HCT116 cells. (A) The protein electropherogram showed the protein expression situation of $N F-\kappa B(p 65), p-N F-\kappa B$ ( $p$-p65), AP-1 (c-jun), p-AP-1 (p-c-jun) and MyD88 in pLKO.1, pLKO.1-sh1 and pLKO.1-sh3 groups in the SW480 cells. (B) The protein expression of the pLKO.1, pLKO.1-sh1 and pLKO.1-sh3 groups was analyzed using a histogram in the SW480 cells. (C) An electropherogram was used to show the protein expression of $N F-\kappa B$ (p65), p-NF- $\kappa B$ (p-p65), AP-1 (c-jun), p-AP-1 (p-c-jun) and MyD88 in pLKO.1, pLKO.1-sh1 and pLKO.1-sh3 groups in the HCT116 cells. (D) The protein expression of the pLKO.1, pLKO.1-sh1 and pLKO.1-sh3 groups was analyzed using a histogram in the HCT116 cells. Error bars represent mean \pm SEM, representative of three experiments. ${ }^{*} \mathrm{P}<0.05,{ }^{* *} \mathrm{P}<0.01,{ }^{* * *} \mathrm{P}<0.001$

findings showed that $M y D 88$ can protect the intestine from tumorigenesis via $I L-18 / M y D 88$ signaling as compared to colitis-associated cancer animal models of $I L-18^{-/-}, I L-18 R 1^{-/-}$, and $M y D 88^{-/-}$mice, as well as wild-type mice (17). By contrast, $M y D 88$ can promote the malignant transformation of colitis to colorectal cancer through the $T L R / M y D 88$ pathway in the $M y D 88$-knockout mice after LPS treatment (24). To date, it remains unclear how and why $M y D 88$ possesses these dual functions in $\mathrm{CRC}$, and the underlying mechanisms require further investigation.
As previously shown, $M y D 88$ signaling plays a role in regulating the growth of normal epithelial and cancer cells in the gut. The activated form of $N F-k B$ is upregulated and functionally correlated with many tumors, modulating proliferation and invasion. AP-1 and, specifically, $c$-Jun, affect tumor cell proliferation, migration and invasion (25). Recent studies have shown that mitogen-activated protein kinases $(M A P K s)$ and nuclear factor- $\kappa \mathrm{B}(N F-\kappa B)$ signaling pathways are two key factors leading to the development of lipopolysaccharide(LPS)-induced acute lung injury (ALI) (26), osteoclastogenesis (27), neuroin- 
A

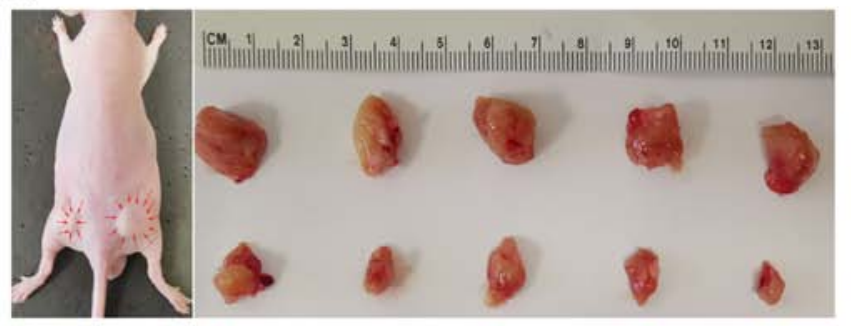

D

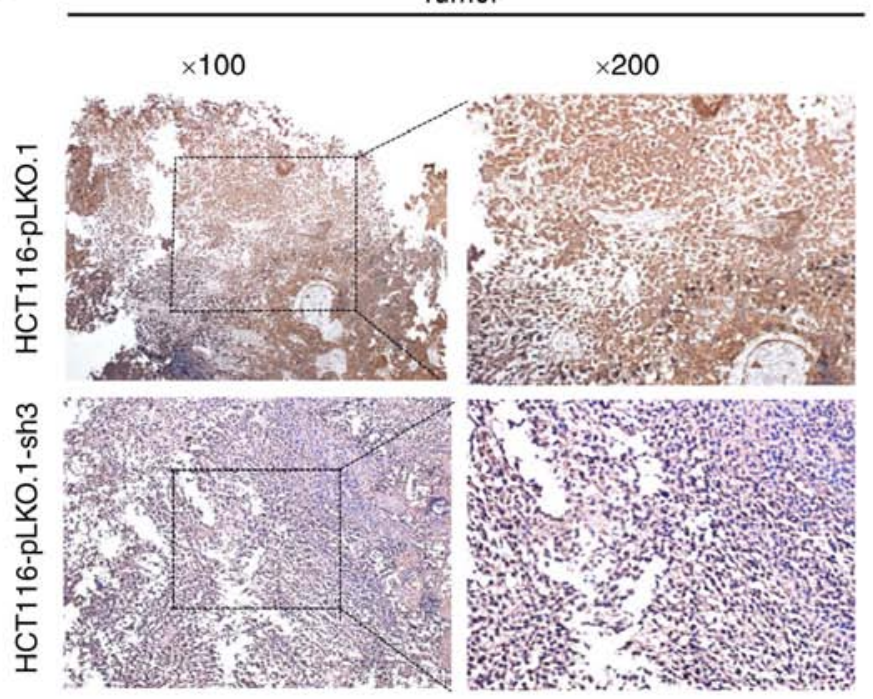

B

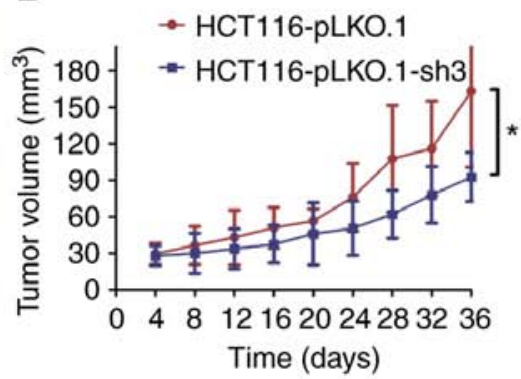

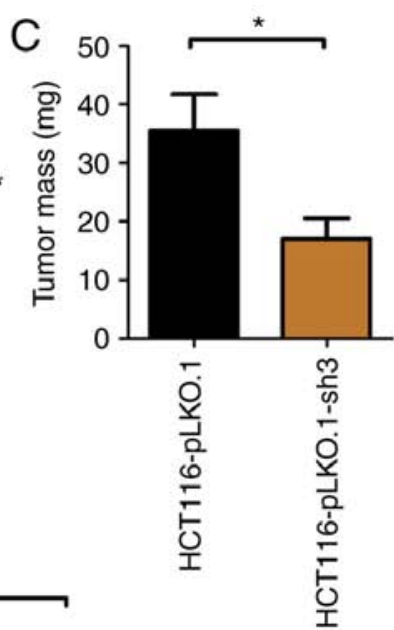

$\mathrm{E}$

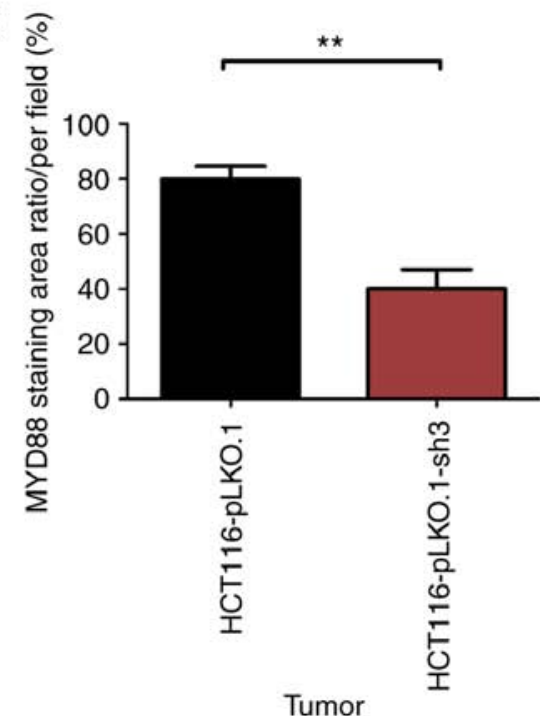

Figure 7. Suppression of tumor growth by silencing the $M y D 88$ gene in the HCT116 cells subcutaneous xenografts. Three groups (pLKO.1 and pLKO.1-sh3 groups) were subcutaneous xenografted onto nude mice. (A) The tumor growth of pLKO.1-sh3 group was significantly inhibited compared with pLKO.1 group. (B) The size of the primary tumors was measured every 4 days. Mice were sacrificed after 5 weeks. (C) The weights of tumors were weighed. Significant differences were identified between the pLKO.1 and pLKO.1-sh3 groups. (D) Immunohistochemical analysis of the expression of $M y D 88$ in HCT116 cell subcutaneous xenograft tumors. Staining showed expression of MyD88 (x100 and x200) in pLKO.1 and pLKO.1-sh3 groups. (E) Quantitative evaluation of the expression of $M y D 88$. Bars show the $M y D 88$ staining area ration/per field. Marked differences between the pLKO.1 and pLKO.1-sh3 groups were identified. ${ }^{*} \mathrm{P}<0.05,{ }^{* *} \mathrm{P}<0.01$.

flammation (28), atherosclerosis (29), tumorigenesis in prostate carcinoma and glioblastoma (25), and activation of human dendritic cells (30). Under the premise of $M y D 88$-induced tumor bioethology, we investigated whether knockdown $M y D 88$ induced changes in $N F-\kappa B$ (p65) and $A P-1$ (MAPKs-c-jun) proteins. In knockdown of $M y D 88$ stably transfected cell lines, we found that $p 65$, phosphorylated $p 65, c$-jun, and phosphorylated $c$-jun protein expression resulted in different degrees of decline. This demonstrates that knockdown of $M y D 88$ gene can affect $M y D 88$-mediated activation of the $N F-\kappa B(p 65)$ and $A P-1$ (MAPKs-c-jun) pathways, thereby effectively retarding the aggressive transformation of the tumor.

In summary, $M y D 88$ could be an independent factor to explore its role in colon cancer, which can be used as a factor in the pathogenesis of colon cancer, and deserves further development as a potential diagnostic and prognostic biomarker. We found that knocking down the $M y D 88$ gene affects proliferation, invasion, and migration of CRC cells, and can reduce the activity of $N F-\kappa B$ and $A P-1$ pathways. These results show that the $M y D 88$ gene plays an important role in promoting CRC and may be exploited as a diagnostic and prognostic biomarker for CRC.

\section{Acknowledgements}

Not applicable.

\section{Funding}

This study was supported by the National Natural Science Foundation of China (grant nos. 81702424 and 81872364), the Fujian Provincial Health Department Young and Middle-aged Talents Training Project (grant no. 2018-ZQN-46), the Joint Funds for the Innovation of Science and Technology, Fujian Province (grant no. 2017Y9092), the Project of Science and Technology Research Program in Fujian Province (grant no. 2016B044), the Fujian Provincial Natural Science Foundation (grant no. 2018J05127), and the National Clinical Key Specialty Construction Project (General Surgery) of China.

\section{Availability of data and materials}

The datasets used or analyzed during the current study are available from the corresponding author on reasonable request. 


\section{Authors' contributions}

JY, GZ, YH and WZ conceived and designed the study. GZ, ZC and CL performed the experiments. SY and JY interpreted the results. GZ and ZC wrote the paper, and all authors contributed to writing. All authors read and approved the final manuscript.

\section{Ethics approval and consent to pariticipate}

The experiment performed with animals was approved by the Institutional Animal Care and Use Committee at the Fujian Medical University.

\section{Patient consent for publication}

Not applicable.

\section{Competing interests}

The authors declare that they have no competing interests.

\section{References}

1. Testa U, Pelosi E and Castelli G: Colorectal cancer: Genetic abnormalities, tumor progression, tumor heterogeneity, clonal evolution and tumor-initiating cells. Med Sci (Basel) 6: E31, 2018.

2. Okugawa Y, Grady WM and Goel A: Epigenetic alterations in colorectal cancer: Emerging biomarkers. Gastroenterology 149: 1204-1225 e12, 2015.

3. Yaghoubi N, Soltani A, Ghazvini K, Hassanian SM and Hashemy SI: PD-1/ PD-L1 blockade as a novel treatment for colorectal cancer. Biomed Pharmacother 110: 312-318, 2019.

4. Marmol I, Sánchez-de-Diego C, Pradilla Dieste A, Cerrada E and Rodriguez Yoldi MJ: Colorectal carcinoma: A general overview and future perspectives in colorectal cancer. Int J Mol Sci 18 : E197, 2017.

5. Lingel A, Ehlers E, Wang Q, Cao M, Wood C, Lin R and Zhang L: Kaposi's sarcoma-associated herpesvirus reduces cellular myeloid differentiation primary-response gene 88 (MyD88) expression via modulation of its RNA. J Virol 90: 180-188, 2016.

6. Feng Y, Zou L, Chen C, Li D and Chao W: Role of cardiac- and myeloid-MyD88 signaling in endotoxin shock: A study with tissue-specific deletion models. Anesthesiology 121: 1258-1269, 2014.

7. Wang H, Li M, Hung CY, Sinha M, Lee LM, Wiesner DL, LeBert V, Lerksuthirat T, Galles K, Suresh M, et al: MyD88 shapes vaccine immunity by extrinsically regulating survival of $\mathrm{CD}^{+} \mathrm{T}$ cells during the contraction phase. PLoS Pathog 12: e1005787, 2016.

8. Xu X, Yin Y, Tang J, Xie Y, Han Z, Zhang X, Liu Q, Qin X, Huang $X$ and Sun B: Long non-coding RNA Myd88 promotes growth and metastasis in hepatocellular carcinoma via regulating Myd88 expression through H3K27 modification. Cell Death Dis 8: e3124, 2017.

9. Syeda S, Patel AK, Lee T and Hackam AS: Reduced photoreceptor death and improved retinal function during retinal degeneration in mice lacking innate immunity adaptor protein MyD88. Exp Neurol 267: 1-12, 2015.

10. d'Adhemar CJ, Spillane CD, Gallagher MF, Bates M, Costello KM, Barry-O'Crowley J, Haley K, Kernan N, Murphy C, Smyth PC, et al: The MyD88+ phenotype is an adverse prognostic factor in epithelial ovarian cancer. PLoS One 9: e100816, 2014.

11. Zhou X, Ramke M, Chintakuntlawar AV, Lee JY, Rajaiya J and Chodosh J: Role of MyD88 in adenovirus keratitis. Immunol Cell Biol 95: 108-116, 2017.

12. Di Padova F, Quesniaux VFJ and Ryffel B: MyD88 as a therapeutic target for inflammatory lung diseases. Expert Opin Ther Targets 22: 401-408, 2018.
13. Reins RY, Courson J, Lema C and Redfern RL: MyD88 contribution to ocular surface homeostasis. PLoS One 12: e0182153, 2017.

14. Zhu G, Zheng W, Huang Y, Hua J, Yang S and Ye J: Expression of MyD88 in cancer tissue of patients with colorectal cancer and clinical significancer. Journal of Jilin University (Medicine Edition) 44: 1047-1051, 2018.

15. Livak KJ and Schmittgen TD: Analysis of relative gene expression data using real-time quantitative PCR and the 2(-Delta Delta C(T)) method. Methods 25: 402-408, 2001.

16. Yan F, Guan J, Peng Y and Zheng X: MyD88 NEDDylation negatively regulates MyD88-dependent NF- $\kappa \mathrm{B}$ signaling through antagonizing its ubiquitination. Biochem Biophys Res Commun 482: 632-637, 2017

17. Tanishima M, Takashima S, Honda A, Yasuda D, Tanikawa T, Ishii $\mathrm{S}$ and MaruYama T: Identification of optineurin as an interleukin-1 receptor-associated kinase 1-binding protein and its role in regulation of MyD88-dependent signaling. J Biol Chem 292: 17250-17257, 2017.

18. Wang L, Yu K, Zhang X and Yu S: Dual functional roles of the MyD88 signaling in colorectal cancer development. Biomed Pharmacother 107: 177-184, 2018.

19. Klimosch SN, Försti A, Eckert J, Knezevic J, Bevier M, von Schönfels W, Heits N, Walter J, Hinz S, Lascorz J, et al: Functional TLR5 genetic variants affect human colorectal cancer survival. Cancer Res 73: 7232-7242, 2013

20. Ikebe M, Kitaura Y, Nakamura M, Tanaka H, Yamasaki A, Nagai S, Wada J, Yanai K, Koga K, Sato N, et al: Lipopolysaccharide (LPS) increases the invasive ability of pancreatic cancer cells through the TLR4/MyD88 signaling pathway. J Surg Oncol 100: 725-731, 2009.

21. Wang EL, Qian ZR, Nakasono M, Tanahashi T, Yoshimoto K, Bando Y, Kudo E, Shimada M and Sano T: High expression of toll-like receptor 4/myeloid differentiation factor 88 signals correlates with poor prognosis in colorectal cancer. Br J Cancer 102: 908-915, 2010.

22. Rakoff-Nahoum S and Medzhitov R: Regulation of spontaneous intestinal tumorigenesis through the adaptor protein MyD88. Science 317: 124-127, 2007.

23. Chang CM, Chia VM, Gunter MJ, Zanetti KA, Ryan BM, Goodman JE, Harris CC, Weissfeld J, Huang WY, Chanock S, et al: Innate immunity gene polymorphisms and the risk of colorectal neoplasia. Carcinogenesis 34: 2512-2520, 2013.

24. Schiechl G, Bauer B, Fuss I, Lang SA, Moser C, Ruemmele P, Rose-John S, Neurath MF, Geissler EK, Schlitt HJ, et al: Tumor development in murine ulcerative colitis depends on MyD88 signaling of colonic $\mathrm{F} 4 / 80^{+} \mathrm{CD} 11 \mathrm{~b}$ (high) Gr1(low) macrophages. J Clin Invest 121: 1692-1708, 2011.

25. Galardi S, Mercatelli N, Farace MG and Ciafre SA: NF- $\kappa B$ and c-Jun induce the expression of the oncogenic miR-221 and miR-222 in prostate carcinoma and glioblastoma cells. Nucleic Acids Res 39: 3892-3902, 2011.

26. Chen X, Yang X, Liu T, Guan M, Feng X, Dong W, Chu X, Liu J, Tian X, Ci X, et al: Kaempferol regulates MAPKs and NF- $\kappa \mathrm{B}$ signaling pathways to attenuate LPS-induced acute lung injury in mice. Int Immunopharmacol 14: 209-216, 2012.

27. Park JW, Yoon HJ, Kang WY, Cho S, Seong SJ, Lee HW, Yoon YR and Kim HJ: G protein-coupled receptor 84 controls osteoclastogenesis through inhibition of NF- $\kappa \mathrm{B}$ and MAPK signaling pathways. J Cell Physiol 233: 1481-1489, 2018.

28. Zheng Y, Fang W, Fan S, Liao W, Xiong Y, Liao S, Li Y, Xiao S and Liu J: Neurotropin inhibits neuroinflammation via suppressing NF-kB and MAPKs signaling pathways in lipopolysaccharide-stimulated BV2 cells. J Pharmacol Sci 136: 242-248, 2018.

29. Pan JX: LncRNA H19 promotes atherosclerosis by regulating MAPK and NF- $\kappa$ B signaling pathway. Eur Rev Med Pharmacol Sci 21: 322-328, 2017.

30. Parola C, Salogni L, Vaira X, Scutera S, Somma P, Salvi V, Musso T, Tabbia G, Bardessono M, Pasquali C, et al: Selective activation of human dendritic cells by OM- 85 through a NF- $\mathrm{KB}$ and MAPK dependent pathway. PLoS One 8: e82867, 2013.

This work is licensed under a Creative Commons Attribution-NonCommercial-NoDerivatives 4.0 International (CC BY-NC-ND 4.0) License. 